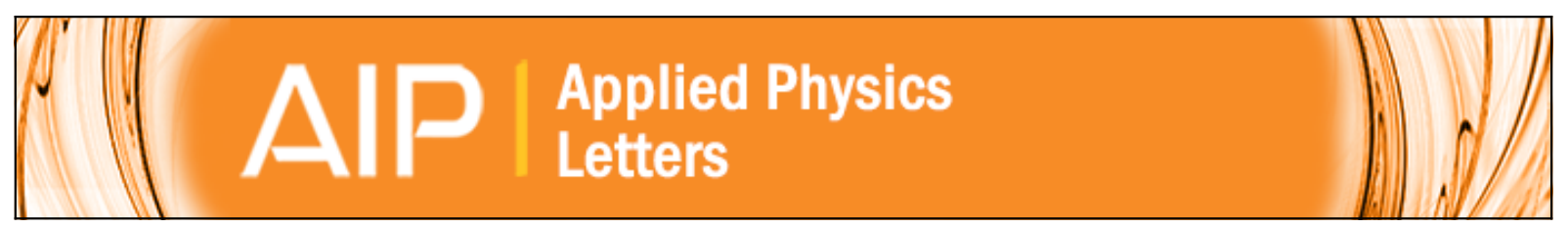

\title{
A nano-indentation study of the contact resistance and resistivity of a bi-layered Au/multi-walled carbon nanotube composite
}

M. P. Down, A. P. Lewis, L. Jiang, and J. W. McBride

Citation: Applied Physics Letters 106, 101911 (2015); doi: 10.1063/1.4915310

View online: http://dx.doi.org/10.1063/1.4915310

View Table of Contents: http://scitation.aip.org/content/aip/journal/apl/106/10?ver=pdfcov

Published by the AIP Publishing

\section{Articles you may be interested in}

Impact of the contact's geometry on the line resistivity of carbon nanotubes bundles for applications as horizontal interconnects

Appl. Phys. Lett. 103, 053115 (2013); 10.1063/1.4817648

An experimental method to determine the resistance of a vertically aligned carbon nanotube forest in contact with a conductive layer

J. Appl. Phys. 112, 044901 (2012); 10.1063/1.4742069

Impact of adsorbed organic monolayers on vacuum electron tunneling contributions to electrical resistance at an asperity contact

J. Appl. Phys. 110, 114307 (2011); 10.1063/1.3664770

Improvement of contact resistance between carbon nanotubes and metal electrodes for high performance electronics

J. Vac. Sci. Technol. B 29, 011011 (2011); 10.1116/1.3520436

Nano- and microscale adhesion energy measurement for Au-Au contacts in microswitch structures

J. Appl. Phys. 100, 104313 (2006); 10.1063/1.2388688

You don't

still use this

cell phone

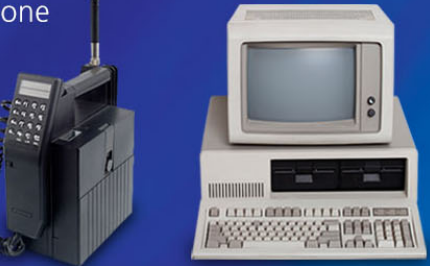

Why are you still using an AFM designed in the 80 's?
It is time to upgrade your AFM

Minimum $\$ 20,000$ trade-in discount for purchases before August 31st

Asylum Research is today's technology leader in AFM 


\title{
A nano-indentation study of the contact resistance and resistivity of a bi-layered Au/multi-walled carbon nanotube composite
}

\author{
M. P. Down, ${ }^{1}$ A. P. Lewis, ${ }^{1}$ L. Jiang, ${ }^{1}$ and J. W. McBride ${ }^{1,2}$ \\ ${ }^{1}$ Faculty of Engineering and the Environment, University of Southampton, Hampshire SO17 1BJ, \\ United Kingdom \\ ${ }^{2}$ University of Southampton Malaysian Campus, Educity Iskandar, Nusajaya, Johor, Malaysia
}

(Received 6 February 2015; accepted 8 March 2015; published online 13 March 2015)

\begin{abstract}
A bi-layer metal-carbon nanotube composite has been developed as a potential low-force electrical contact surface, for application in micro-electromechanical systems switching devices. The samples consist of a vertically aligned forest of multi-walled carbon nanotube (MWCNT), sputter coated with a layer of Au. The effect of varying the components and composition are investigated by means of a modified nano-indenter. By measuring the contact resistance of the composites under various loading conditions, the electrical properties and performance can be evaluated. The composites are shown to have homogenous properties, with each of the layers influencing the total electrical characteristics of the samples. The internal structure of the sample, the MWCNT height and penetration of gold into the forest is shown to directly influence the performance and characteristics of the samples. By analyzing the samples as bulk, the effective resistivities of the composites are also determined to have values from $303 \mathrm{n} \Omega \mathrm{m}$ down to $54 \mathrm{n} \Omega \mathrm{m}$, depending on the composition of the samples. (C) 2015 AIP Publishing LLC. [http://dx.doi.org/10.1063/1.4915310]
\end{abstract}

Since their discovery the mechanical properties of carbon nanotubes (CNTs) have attracted attention in both theoretical and experimental studies. Having both high elastic modulus and high strength, ${ }^{1-4}$ CNTs are largely considered to be superior to conventional carbon fibers. They have also been shown to exhibit excellent thermal and electrical conductivity, due to their nanoscale dimensions and ballistic electronic conduction. ${ }^{5}$ Based on these attributes, CNTs provide the potential to manufacture composites of metals with the CNTs as a reinforcement component ${ }^{4-6}$ and the potential to manufacture composites consisting of a metal matrix has been frequently investigated. In existing metal-CNT composites, the properties are largely characterized by an increase in the strength and elasticity, due to the presence of the CNTs, whilst the electrical and thermal conductivity are generally the same as or close to the properties of the pure metal matrix. ${ }^{5}$ The combination of mechanical high elasticity and high electrical conductivity that such metal-CNT composites exhibit, provide properties for applications in microelectromechanical systems (MEMS), where the high elasticity provide high lifetimes in mechanical components, and the high conductivity provides good electrical performances. Despite this, they are mostly used or designed for larger structural applications. ${ }^{7}$ A unique metal carbon nanotube composite is introduced, which utilizes the mechanical properties of CNTs in a different manner to most metal-CNT composites. Vertically aligned multi-walled CNTs (MWCNTs) provide a compliant subsurface, whilst a sputtered metal component, in this case gold, provides electrical conductivity.

The composite has shown potential as a long-lifetime switching surface under MEMS switching contact conditions. ${ }^{8-10}$ Studies of the Au/MWCNT composites compare to the more orthodox sputtered $\mathrm{Au} / \mathrm{Si}$ as an electrical contact, showing the metal-CNT composite to have exceeded the lifetime of the orthodox contact by several orders of magnitude. ${ }^{8-10}$ In this study, the results are used to evaluate the electromechanical properties of the samples by investigating the contact resistance of the composite under various loads by means of a modified nanoindenter and fitting to the Holm electrical contact model. Holm described the contact resistance of a pair of contacts in terms of the applied load, $\mathrm{F}$, as

$$
R_{c}=\left(\frac{\rho^{2} \eta \pi H}{4 F}\right)^{\frac{1}{2}}
$$

where $\rho$ is the electrical resistivity of the contact materials, $\mathrm{H}$ is the material hardness, and $\eta$ is a coefficient used to describe the influence of contamination or insulating films which reduce the conducting contact area, in turn increasing the contact resistance. From this an evaluation of the resistivity and reduction coefficient, $\rho \sqrt{\eta}$, can be made in terms of the composition, evaluating the performance of the composites as electrical contacts and for electromechanical applications. The application of this model assumes that the contacting asperities deform plastically, relating the real contact area, $A$, and the applied load by means of the material hardness according to $F=H A$. Also that the samples hardness are uniform and do not vary with depth ${ }^{11}$ and that the samples conduct without the influence of a constriction resistance related to thin film conduction, acting as homogenous bulk samples.

For the Au/MWCNT composite, a forest of MWCNTs was grown onto a $\mathrm{Si}$ wafer using a thermal CVD process. A buffer layer of $\mathrm{Al}_{2} \mathrm{O}_{3}, 1.5 \mathrm{~nm}$ thick, was sputtered, followed by a catalyst layer of $\mathrm{Fe}, 10 \mathrm{~nm}$ thick, which promotes the growth of CNTs. We assume the packing density of MWCNTs to be constant, as it is assumed to be controlled by the catalyst and annealing conditions during the sample 
TABLE I. Samples generated: Exact measured thicknesses and measured mechanical properties.

\begin{tabular}{|c|c|c|c|c|c|}
\hline \multirow[b]{2}{*}{ Sample No. } & \multicolumn{5}{|c|}{ Sample composition } \\
\hline & $\begin{array}{c}\text { Composition } \\
{[\mathrm{Au}(\mathrm{nm}) / \mathrm{MWCNT}(\mu \mathrm{m})]}\end{array}$ & Thickness ratio $^{\mathrm{a}}$ & Measured $\mathrm{Au}(\mathrm{nm})^{\mathrm{b}}$ & Measured MWCNT $(\mu \mathrm{m})^{\mathrm{b}}$ & Sample hardness $(\mathrm{MPa})^{\mathrm{c}}$ \\
\hline 1 & $300 / 50$ & 0.006 & 301 & 51.31 & 0.282 \\
\hline 2 & $500 / 30$ & 0.017 & 498 & 29.87 & 1.055 \\
\hline 3 & $500 / 50$ & 0.010 & 500 & 50.01 & 0.639 \\
\hline 4 & $500 / 80$ & 0.006 & 499 & 80.41 & 0.035 \\
\hline 5 & $800 / 50$ & 0.016 & 799 & 50.02 & 2.8933 \\
\hline
\end{tabular}

${ }^{\mathrm{a}}$ Defined as the ratio of the Au thickness (in nm) and the MWCNT layer (in nm).

${ }^{\mathrm{b}}$ Measured by laser surface profiler, with vertical resolution of $10 \mathrm{~nm}$.

${ }^{\mathrm{c}}$ Evaluated by nanoindentation at $0.25-0.75 \mathrm{mN}$, sample stiffness is evaluated as the effective Young's modulus of the composites. ${ }^{11}$

development, which were held constant at a temperature of $900{ }^{\circ} \mathrm{C}$ for $10 \mathrm{~min}$.

The samples investigated were of the same structure as those tested for potential in MEMS switching applications, ${ }^{8-10}$ consisting of a subsurface of MWCNTs sputter coated with a conductive Au film. In order to further investigate the potential applications of the composite, a series of samples with varying composition were fabricated for this study, these were composed of different thickness of Au film and lengths of MWCNTs. A sample matrix of the varied compositions was generated in order to characterize the influence of the components on the electromechanical performance of the surface, and to optimize the composition for applications under MEMS contact conditions. By varying the thickness of the Au and the height of the MWCNT layers, the influence of each could be determined. These samples are compared to a control sample of $500 \mathrm{~nm}$ Au sputter coated onto a Si substrate, with a $10 \mathrm{~nm} \mathrm{Cr}$ adhesion layer, which is a commonly used contact surface in MEMS contact devices.

Three sets of MWCNT lengths were grown; $30 \mu \mathrm{m}$, $50 \mu \mathrm{m}$, and $80 \mu \mathrm{m}$, by increasing the growth time in the CVD growth process. For height measurements, a patterned sample was generated to give an area where surface height can be compared to the Si wafer, which can be accurately measured using a laser surface profiler. To avoid any edge effects on the growth of the CNTs, heights were measured at three points at the center of the samples and averaged for accuracy. The same process was carried out to measure the thickness of the deposited Au; a sacrificial Si chip was placed in the sputtering chamber during the MWCNT coating. Three $50 \mu \mathrm{m}$ MWCNT substrates were coated with an Au film of thickness: $300 \mathrm{~nm}, 500 \mathrm{~nm}$, and $800 \mathrm{~nm}$, and two substrates $30 \mu \mathrm{m}$ and $80 \mu \mathrm{m}$ MWCNT forests were coated with $500 \mathrm{~nm}$
$\mathrm{Au}$, generating a matrix of 5 compositions of Au/MWCNT samples, shown in Table I.

In order to achieve the low forces required with repeatability and stability, a modified nano-indenter was utilized. The nano-indenter provided the controlled mechanical loading required whilst the addition of a data acquisition module allowed for the application of a controlled electrical current ( $100 \mathrm{~mA}$ for this study), and measurement of the voltage drop. The standard diamond contact tip was replaced by a stainless steel hemispherical contact, $2 \mathrm{~mm}$ in diameter, which was coated with a $10 \mathrm{~nm} \mathrm{Cr}$ adhesive layer and a $500 \mathrm{~nm} \mathrm{Au}$ layer. This modified tip allowed for a four-wire measurement arrangement to be applied to measure the contact resistance illustrated in Figure 1(b) and is consistent with previous experimental studies. ${ }^{12}$ By using the specialized indenter tip, the compliant effect of the MWCNT composite surface is maximized as it requires the composite to conform to the shape of the hemisphere. Whilst using this system the force and contact resistance could be measured simultaneously, the contact resistance was evaluated in post processing from the applied current and voltage drop.

Each sample was indented with 10 different loads from $0.2 \mathrm{mN}$ to $2 \mathrm{mN}$, each indent was at a new surface location, separated by $500 \mu \mathrm{m}$, and each load repeated 10 times for consistency at new surface locations. The loading rates were held constant and the maximum load was held constant for $30 \mathrm{~s}$ during each indent. The properties of the contacts were only evaluated during this static stage, of the indentation to allow the surfaces to settle and prevent loading and surface anomalies, such as oxide layers or contamination, from effecting results.

The results are presented in terms of the contact resistance under various mechanical loads, shown in Figure 2(a),
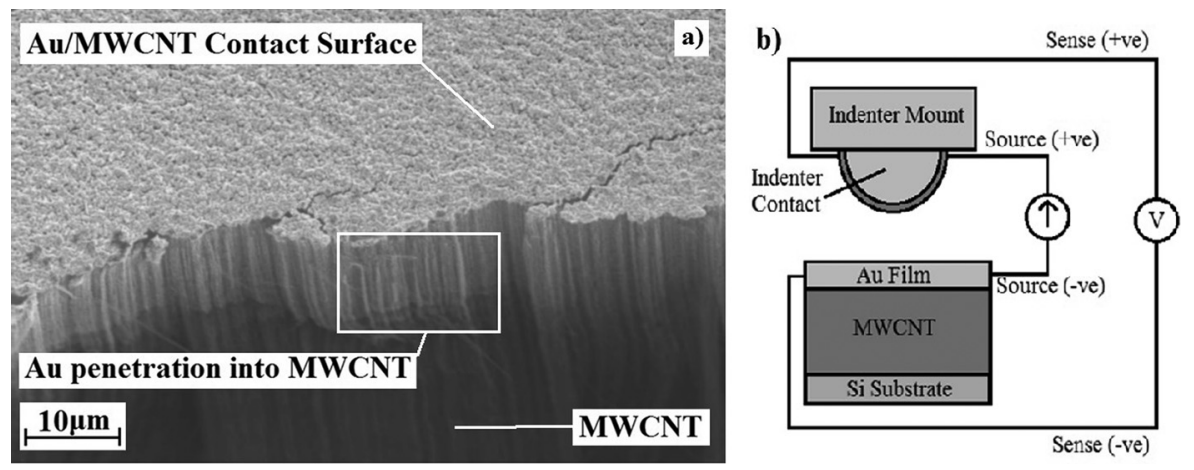

FIG. 1. (a) Typical structure of the Au/ MWCNT composites, in this case $500 \mathrm{~nm} \mathrm{Au}$ coating on a $50 \mu \mathrm{m}$ MWCNT subsurface, where the Au penetrates into the MWCNT subsurface; (b) four-wire measurement arrangement for the contact resistance measurement system integrated into the nanoindentation. The current source and contact voltage drop are supplied and measured by a data acquisition module. 

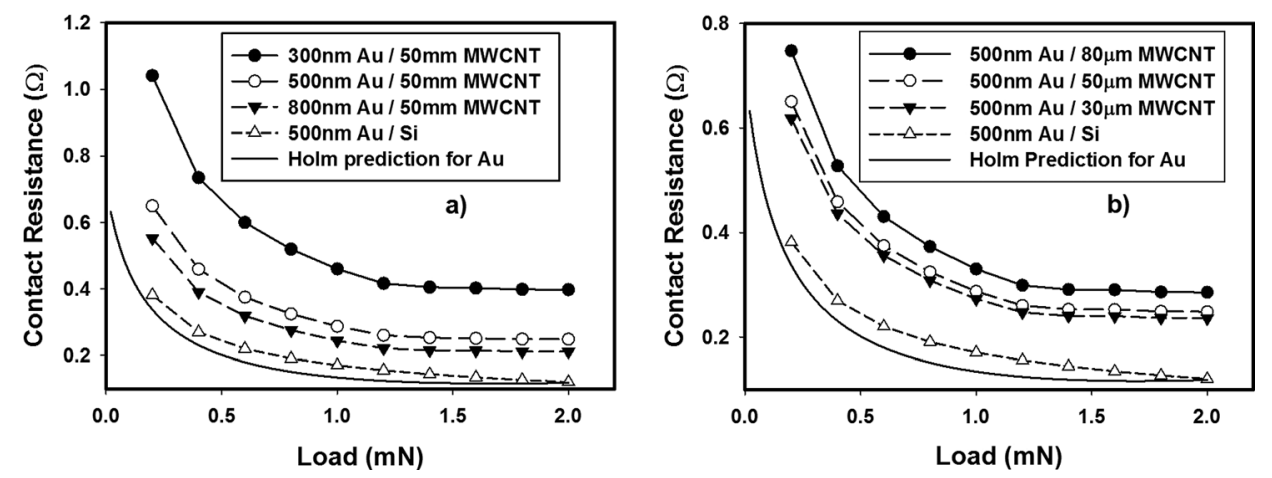

FIG. 2. Contact resistance for various loads and compositions (a) illustrates the effects of changing the Au layer thickness and (b) the height of the MWCNT subsurface. which shows the effects of varying the Au layer thickness on the contact resistance, and Figure 2(b), which shows the effect of altering the height of the MWCNT forest. The contact resistances observed are mostly below $1 \Omega$. There is only one instance of this limit being exceeded, for the $300 \mathrm{~nm} \mathrm{Au} / 50 \mu \mathrm{m}$ MWCNT sample where the load was only $0.2 \mathrm{mN}$, which was the lowest load applied to the sample that clearly represents the worst conductive properties of the samples investigated. The trend of the results matches with those in the Holm model, Eq. (1), and the $\mathrm{Au} / \mathrm{Si}$ sample, shown in Figure 2, albeit with higher contact resistances. This behavior indicates that the samples behave as single layer bulk samples and not multilayered thin film composites. This is reinforced in Figure 3, by showing that the square of the contact resistance is proportional to the inverse of the contact force for all samples. The slight increase in the observed resistance between the Au/Si sample and the Holm prediction for $\mathrm{Au}$ can be attributed to the influence of thin film conduction, ${ }^{12}$ while the results for the various $\mathrm{Au} /$ MWCNT composites indicate the influence of each of the bilayer components. The influence of the gold layer is clearly significant in controlling the electromechanical performance of the composite. As the vertically aligned MWCNT forest conductivity in the lateral direction (perpendicular to the growth direction) is poor; ${ }^{13}$ the composite depends on the gold layer to conduct laterally.

Increasing the carbon nanotube subsurface layer height has been reported to be decrease the hardness of the composite, ${ }^{11}$ and as shown in Table I, hence increasing the contact area under a given load. The Holm model predicts that for a softer material the contact resistance should be reduced. Yet what was observed in Fig. 2(b), was that the increase in the MWCNT subsurface height increased the contact resistance. This suggests that the increase in MWCNT height changes the structure of the composite and how the $\mathrm{Au}$ penetrates into the MWCNT subsurface, thereby affecting the efficiency of the lateral conduction of the composite. Figure 2(b) demonstrates that the presence of the MWCNTs directly influence the electrical properties, increasing the effective resistivity of the composite. This has been further investigated further by fitting the experimental values of the contact resistance to the Holm model, shown in Figures 3(a) and 3(b) for varying thicknesses of Au and heights of MWCNT composites, respectively.

By analyzing the contact resistance results and fitting to the Holm model, the effective resistivity can be evaluated. The results of which are shown in Figure 4, for simplicity the samples are defined in terms of the thickness ratio as defined in Table I. These values, although not the definitive resistivity values, do provide a practical evaluation of the performance of the contact surface as electrical contacts. If the samples are assumed to have the resistivity of the $\mathrm{Au}$ film, this then becomes an analysis of the reduction coefficient, and hence the efficiency of these composites when forming contacts. However, it can be assumed that the surfaces are clean and that varying the components will affect the resistivity of the composites. A general trend can be observed where these properties can be used to define the electrical performance of the composites; as the thickness ratio increases the effective resistivity appears to tend towards the value for the $\mathrm{Au} / \mathrm{Si}$ sample.

As expected, increasing the thickness of the Au layer decreased the resistivity of the composite significantly. However, the trend shown in Figure 4, again, demonstrates that an increase in MWCNT height results in an increase in contact resistance. There are a number of potential causes for this unexpected behavior. First, as shown in Figure 1(a), the $\mathrm{Au}$ is known to penetrate into the MWCNT subsurface. The depth of this layer and the structure that results is not clearly defined, and the height of the MWCNTs potentially
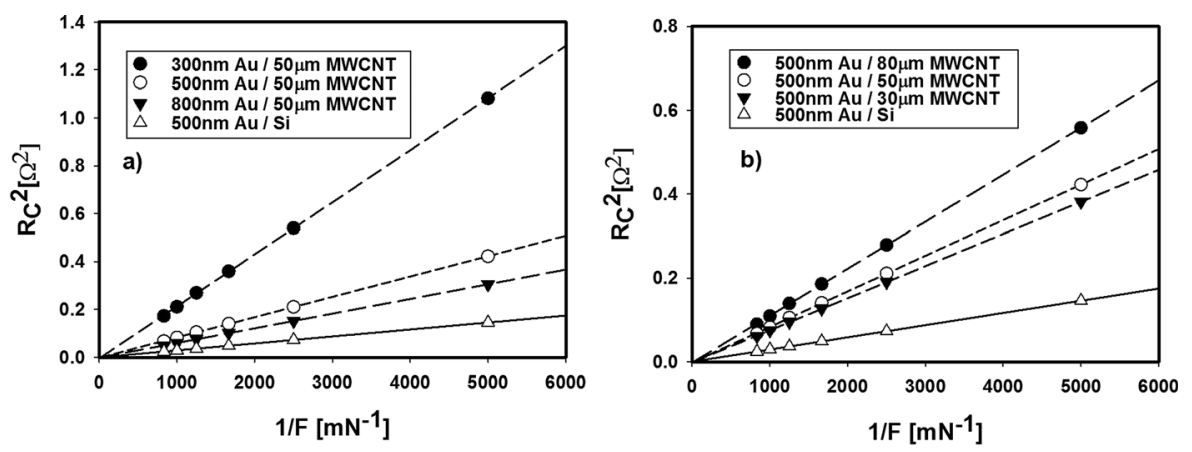

FIG. 3. Fitting the experimental contact resistance to the linear form of the Holm model for (a) various Au thickness and (b) various MWCNT heights for loads $1-2 \mathrm{mN}$. 


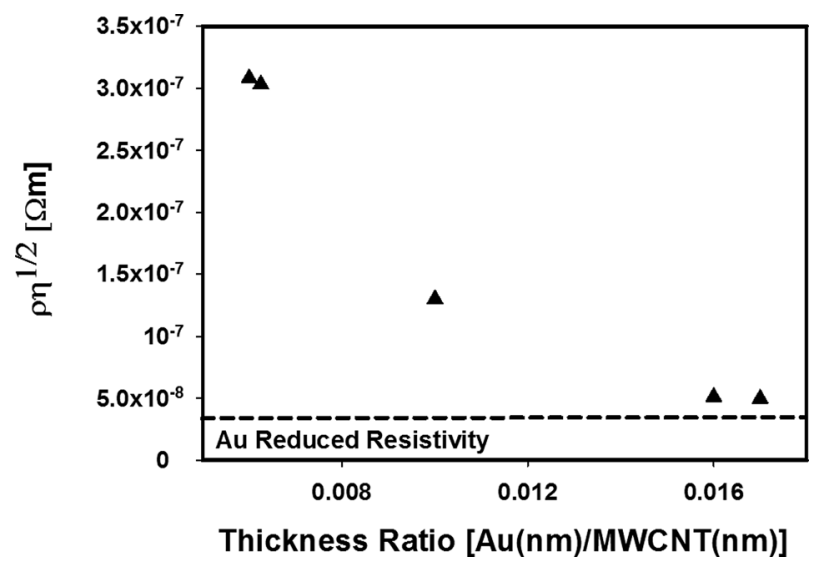

FIG. 4. Effective electrical resistivity of the samples evaluated by fitting the Holm model to the contact resistance measurements. As a result, the values include any effects of the oxide and contamination layers described by the reduction coefficient, $\eta$. The dotted line represents the value from analysis of the $\mathrm{Au} / \mathrm{Si}$ sample.

enables a deeper Au penetration thereby reducing the efficiency of the conductivity in the lateral direction. It is possible that the length of the CNT could influence the depth of penetration of the Au layer. This aspect of the surface has been presented by Liu et al., ${ }^{14}$ but requires further investigation. In addition, an increase in the MWCNT height would result in a decrease in surface hardness thereby allowing the surface to comply more efficiently with the incoming contact, resulting in an increase in the nominal contact area and number of contact spots.

The results demonstrate a high level of consistency in the samples, consistently providing a contact resistance below $1 \Omega$, for even the lowest loads tested, with one exception in the case of the $300 \mathrm{~nm} \mathrm{Au} / 50 \mu \mathrm{m}$ MWCNT sample. This either provides a lower limit the forces in application or could be addressed by reducing the MWCNT thickness layer if a $300 \mathrm{~nm} \mathrm{Au}$ film is required. The experiment setup has been designed to accurately evaluate the electrical contact properties of the composite and has shown potential for analysing the contact properties under low loads. In previous studies, ${ }^{7}$ it was shown that it was not valid to consider the conductivity of the Au/MWCNT to be similar to that of an $\mathrm{Au}$ film. The results here demonstrate that the MWCNT subsurface is not just a compliant subsurface contributing mechanically but significantly contributes to the electrical properties. Studies of the structure of MWCNT forests have shown that, due to the growth mechanisms, the density of the CNTs is higher at their base then at their peak. ${ }^{7}$ The reduced density with height and evidence of the penetration of the sputtered $\mathrm{Au}$ into the CNT subsurface, shown in Figure 1, suggest that the increase in CNT height influences the depth of penetration of the sputtered layer, and consequently the conductive properties of the composite. By investigating the effect of the composition of the composite in this study, the effective resistivity of the composite, and the efficiency of the composite as an electrical contact has been ranked in terms of the composition. It has been deduced that the resulting electrical properties are strongly dominated by the internal structure of the composite.
To summarize, an improved four-wire measurement system and modified nanoindentation rig have been developed to improve the understanding of the electromechanical properties of Au/MWCNT composites. The samples tested have shown a level of consistency in their performance, where the contact resistance is mostly less than $1 \Omega$. Whilst the addition of the MWCNTs had been shown to greatly improve the lifetime of the composite in switching tests, the apparent reduction in conductivity with their inclusion raises further questions of the interior structure of the composites. The trends observed suggest that by controlling the Au thickness and MWCNT height it is possible to control the electrical and mechanical properties of the composite. An understanding of the effects of the varying the composite parameters is significantly important for optimizing the composite. It has been shown conclusively that the presence of MWCNTs impacts the resistive properties of the sample, which can be defined in terms of the thickness ratio. The increase of which tends the properties towards those of the $\mathrm{Au} / \mathrm{Si}$ sample.

The Holm Model, despite not often being applied for low contact forces due to lack of inclusion of ballistic electron transport, has proven useful for analysis of the effective electrical properties of the samples. Whilst these values are not definitive properties, they provide an analysis of the effectiveness of the composites for use as electrical switching surfaces. If the resistivity of the samples is assumed to be that of gold then the analysis is that of the reduction coefficient, and how efficiently the samples form a contact. A combination of the results presented in the paper with lifetime testing of the composites will yield an optimized solution for Au-MWCNT electrical switching contacts; where the trade-offs for the sample compositions can be balanced.

${ }^{1}$ J.-P. Salvetat, G. Andrew, D. Briggs, J.-M. Bonard, R. R. Bacsa, A. J. Kulik, T. Stöckli, N. A. Burnham, and L. Forró, "Elastic and shear moduli of single-walled carbon nanotube ropes,” Phys. Rev. Lett. 82, 944 (1999).

${ }^{2}$ M.-F. Yu, O. Lourie, M. J. Dyer, K. Moloni, T. F. Kelly, and R. S. Ruoff, "Strength and breaking mechanism of multiwalled carbon nanotubes under tensile load," Science 287, 637 (2000).

${ }^{3}$ P. Poncharal, Z. L. Wang, D. Ugarte, and W. A. de Heer, "Electrostatic deflections and electromechanical resonances of carbon nanotubes," Science 283, 1513 (1999).

${ }^{4}$ A. Krishnan, E. Dujardin, T. W. Ebbesen, P. N. Yianilos, and M. M. J. Treacy, "Young's modulus of single-walled nanotubes," Phys. Rev. B 58, 14013 (1998).

${ }^{5}$ A. K.-T. Lau and D. Hui, "The revolutionary creation of new advanced materials-Carbon nanotube composites," Composites, Part B 33, 263-277 (2002).

${ }^{6}$ C. L. Xu, B. Q. Wei, R. Z. Ma, J. Liang, X. K. Ma, and D. H. Wu, "Fabrication of aluminum-carbon nanotube composites and their electrical properties," Carbon 37, 855-858 (1999).

${ }^{7}$ S. R. Bakshi, D. Lahiri, and A. Agarwal, "Carbon nanotube reinforced metal matrix composites-A review," Int. Mater. Rev. 55, 41-47 (2010).

${ }^{8}$ J. W. McBride, L. Jiang, and C. Chianrabutra, "Fine transfer in electrical switching contacts using gold coated carbon-nanotubes," in Joint Conference of 26th International Conference on Electrical Contacts and 4th International Conference on Reliability of Electric Products and Electric Contacts, Beijing, 2012, pp. 353-358.

${ }^{9}$ A. P. Lewis, M. P. Down, C. Chianrabutra, L. Jiang, S. M. Spearing, and J. W. McBride, "The effect on switching lifetime of chromium adhesion layers in gold-coated electrical contacts under cold and hot switching conditions," in 59th IEEE Holm Conference on Electrical Contacts, Newport, RI, 2013, pp. 49-55.

${ }^{10}$ J. W. McBride, E. M. Yunus, and S. M. Spearing, "Improving the contact resistance at low force using gold coated carbon nanotube surfaces," Eur. Phys. J.: Appl. Phys. 50, 507-513 (2010). 
${ }^{11}$ M. P. Down, R. Cook, L. Jiang, and J. W. McBride, "Mechanical characterisation and optimisation of carbon nanotube composite surfaces," in International Conference on Electrical Contacts, Dresden, 2014, pp. 1-6.

${ }^{12}$ E. M. Yunus, S. M. Spearing, and J. W. McBride, "The relationship between contact resistance and contact force on Au coated carbon nanotube surfaces under low force conditions," IEEE Trans. Compon. Packag. Technol. 32(3), 650-657 (2009).
${ }^{13}$ E. Van Hooijdonk, C. Bittencourt, R. Snyders, and J.-F. Colomer, "Functionalization of vertically aligned carbon nanotubes," J. Nanotechnol. 4, 129-152 (2013).

${ }^{14}$ H. Liu, J. W. McBride, S. H. Pu, M. P. Down, and L. Jiang, "Mechanical characterization of a Au coated carbon nanotube multilayered structure," in 60th IEEE Holm Conference on Electrical Contacts, New Orleans, USA, 12-15 October 2014. 